\title{
High Modulus Regenerated Cellulose Fibers Spun from a Low Molecular Weight Microcrystalline Cellulose Solution
}

Chenchen Zhu, ${ }^{\dagger}$ Robert M. Richardson, ${ }^{\ddagger}$ Kevin D. Potter, ${ }^{\dagger}$ Anastasia F. Koutsomitopoulou, ${ }^{\dagger}$ Jeroen S. van Duijneveldt, ${ }^{\S}$ Sheril R. Vincent, ${ }^{\dagger}$ Nandula D. Wanasekara, ${ }^{\|}$Stephen J. Eichhorn, ${ }^{*}, \|$ and Sameer S. Rahatekar*, ${ }^{*}$

${ }^{\dagger}$ Advanced Composites Centre for Innovation and Science (ACCIS), Department of Aerospace Engineering, University of Bristol, Queen's Building, University Walk, Bristol BS8 1TR, U.K.

${ }^{\ddagger} \mathrm{HH}$ Wills Physics Laboratory, Physics Department, University of Bristol, Tyndall Avenue, Bristol BS8 1TL, U.K.

${ }^{\S}$ School of Chemistry, University of Bristol, Cantock's Close, Bristol BS8 1TS, U.K.

${ }$ College of Engineering, Maths and Physical Sciences, University of Exeter, Stocker Road, Exeter EX4 4QL, U.K.

\section{Supporting Information}

ABSTRACT: We have developed a novel process to convert low molecular weight microcrystalline cellulose into stiff regenerated cellulose fibers using a dry-jet wet fiber spinning process. Highly aligned cellulose fibers were spun from optically anisotropic microcrystalline cellulose/1-ethyl-3-methylimidazolium diethyl phosphate (EMImDEP) solutions. As the cellulose concentration increased from 7.6 to $12.4 \mathrm{wt} \%$, the solution texture changed from completely isotropic to weakly nematic. Higher concentration solutions (>15 wt \%) showed strongly optically anisotropic patterns, with clearing temperatures ranging from 80 to $90{ }^{\circ} \mathrm{C}$. Cellulose fibers were spun from $12.4,15.2$, and 18.0 wt $\%$ cellulose solutions. The

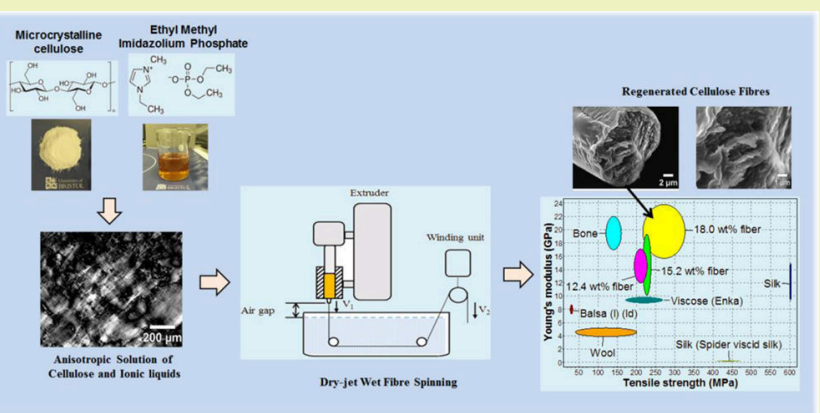
physical properties of these fibers were studied by scanning electron microscopy (SEM), wide angle X-ray diffraction (WAXD), and tensile testing. The $18.0 \mathrm{wt} \%$ cellulose fibers, with an average diameter of $\sim 20 \mu \mathrm{m}$, possessed a high Young's modulus up to $\sim 22 \mathrm{GPa}$, moderately high tensile strength of $\sim 305 \mathrm{MPa}$, as well as high alignment of cellulose chains along the fiber axis confirmed by X-ray diffraction. This process presents a new route to convert microcrystalline cellulose, which is usually used for low mechanical performance applications (matrix for pharmaceutical tablets and food ingredients, etc.) into stiff fibers which can potentially be used for high-performance composite materials.

KEYWORDS: Microcrystalline cellulose, Ionic liquid, Anisotropy, Fiber spinning, Alignment, Mechanical property

\section{INTRODUCTION}

Cellulose is a polysaccharide consisting of long linear chains of $\beta$ - $(1,4)$-D-glucose units, ${ }^{1}$ which is the most abundant and renewable polymer in the world with an annual biosphere production about $90 \times 10^{9}$ metric tons. ${ }^{1}$ As a biopolymer, it possesses desirable mechanical properties, ${ }^{1}$ such as high molecular order, as well as excellent renewability, biodegradability, and biocompatibility. ${ }^{2}$ However, the multiple hydroxyl groups on cellulose form intra- and inter-molecular, hydrogen bonding, holding the chains firmly together side-by-side thus making it relatively insoluble in most traditional solvents such as water, ethanol, and acids. ${ }^{1,3}$ In addition, hydrophobic interactions may contribute to this insolubility. ${ }^{4}$

The viscose and lyocell processes are the two main methods for manufacturing regenerated cellulose fibers. The viscose process requires multiple manufacturing steps including the use of chemical derivatization using high aggressive solvents such as sodium hydroxide and carbon disulfide. This makes the process more expensive and environmentally hazardous. ${ }^{5}$ The lyocell process (which uses $\mathrm{N}$-methylmorpholine $\mathrm{N}$-oxide, $\mathrm{NMMO}$, as a solvent) has been proposed as an alternative to the viscose process. The solvent used in this process is thermally unstable and requires significant financial investment in safety technology. Over the past decade, a new generation of solvents for cellulose called ionic liquids (ILs) have attracted much attention for their chemical and thermal stabilities, ${ }^{6}$ high decomposition points, low vapor pressures, low flammability, excellent recoverability (>99.5\%), and reusability. ${ }^{8}$ The anions in ILs can form hydrogen bonds with hydroxyl hydrogen and oxygen atoms in cellulose, which break down the hydrogen bonding network thus contributing to the dissolution of cellulose. $^{3}$

Unlike the viscose process, the ionic liquids can dissolve cellulose in one-step without the need for chemical

Received: March 19, 2016

Revised: June 25, 2016

Published: July 22, 2016 
derivatization. They are thermally more stable than the NMMO used in the lyocell process and the filaments used do not fibrillate. Ionic liquids are expensive solvents, but due to their less aggressive nature (unlike solvents used in the viscose and lyocell processes), their ability to dissolve cellulose in one step, and their higher thermal stability, they do not require large financial investment in safety and auxiliary equipment. They can be easily recycled with high recycling efficiency. Hence they can potentially be an attractive alternative to the traditional viscose process in spite of their high initial cost.

Recently, some researchers have reported the formation of optically anisotropic solutions by dissolving cellulose in ILs. ${ }^{9,10}$ It is well-known that anisotropic solutions can significantly improve the spinnability of fibers; ${ }^{11}$ the regenerated fibers produced from such solutions possess excellent mechanical properties due to their intrinsic, highly oriented, and rigid molecular backbones as well as strong intermolecular hydrogen bonds. ${ }^{12}$ Northolt et al. produced very good quality cellulose fibers from an anisotropic solution of cellulose in phosphoric acid with a modulus of $44 \mathrm{GPa}$ and tensile strength of 1.7 GPa. ${ }^{13}$ However, a relatively complex mixing process was used in their study, involving orthophosphoric acid, pyrophosphoric acid, polyphosphoric acid, phosphorus pentoxide, and water.

In this study, a low molecular weight microcrystalline cellulose, which is usually used for applications requiring low mechanical properties (medical tablets, food-stuffs, etc.), is used to spin fibers with a high tensile modulus from optically anisotropic solutions, using a phosphate-based ionic liquid as a solvent. The concentration of microcrystalline cellulose dissolved in the ionic liquid was optimized to achieve optically anisotropic solutions to improve the alignment of cellulose chains. Similarly, a high fiber extrusion/winding ratio was used to further improve this alignment to obtain high modulus cellulose fibers. The manufacturing process established in this study has great potential to produce regenerated cellulose fibers for the composites industry by introducing sustainable and renewable fibers with enhanced mechanical performance.

\section{EXPERIMENTAL METHODS}

Materials and Dissolution Method. Highly pure microcrystalline cellulose (MCC), VIVAPUR101, with a viscosity-averaged degree of polymerization (DP) between 200 and 220,9,14 was purchased from JRS Pharma GmbH \& Co. KG (Rosenberg, Germany). Ionic liquid (IL) 1-ethyl-3-methylimidazolium diethyl phosphate (EMImDEP) was purchased from Sigma-Aldrich (713392, Gillingham, UK). A magnetic stirrer hot plate (Fisher Scientific, Loughborough, UK) with an oil bath was used for the preparation of cellulose solutions. The dissolution process was carried out in a fume hood. 7.6 (2.3), 12.4 (3.7), 15.2 (4.6), and $18.0 \mathrm{wt} \%$ (5.4 g) of cellulose were added to $30 \mathrm{~g}$ EMImDEP and heated at $95{ }^{\circ} \mathrm{C}$ with magnetic stirring at $100 \mathrm{rpm}$ for $24 \mathrm{~h}$, respectively.

Characterization of Cellulose/EMImDEP Solutions. Small amounts of 7.6, 12.4, 15.2, and 18.0 wt \% cellulose/EMImDEP solutions were pressed to form thin films between two glass slides. ${ }^{14}$ These films were placed on a Linkam PE120 thermoelectrically controlled stage connected to an EHEIM professional 3 water filter for cooling. The observation of optical anisotropy and clearing temperatures of cellulose solution films was conducted using an Olympus BX51 polarized optical microscope with a PixeLINK PL-B625 camera with a $\times 10$ objective. The films were heated at a heating rate of 10 ${ }^{\circ} \mathrm{C} / \mathrm{min}$ from 25 to $90{ }^{\circ} \mathrm{C}\left( \pm 0.1{ }^{\circ} \mathrm{C}\right)$, maintaining a fixed temperature for 5 min before taking the polarized optical micrographs. Micrographs were taken at $25{ }^{\circ} \mathrm{C}$ first, then at $10^{\circ} \mathrm{C}$ increments from 30 to $60{ }^{\circ} \mathrm{C}$, and then at $5{ }^{\circ} \mathrm{C}$ increments from 60 to $90{ }^{\circ} \mathrm{C}$ (see Supporting Information Figures S1-3).
Fiber Spinning of Cellulose. Specifically designed fiber spinning equipment (Rondol, UK), which consists of a vertical ram extruder, a water bath, and a haul-off unit, was used for the dry-jet wet fiber spinning of the regenerated cellulose fibers (Figure 1).

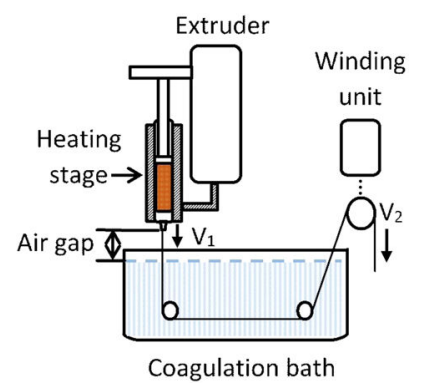

Figure 1. Schematic of dry-jet wet fiber spinning process for cellulose fiber with constant extrusion velocity $\left(V_{1}\right)$ and winding velocity $\left(V_{2}\right)$.

The 7.6, 12.4, 15.2, and 18.0 wt \% cellulose/EMImDEP solutions were transferred into a removable extruder barrel and degassed in a vacuum oven at $80{ }^{\circ} \mathrm{C}$ for $18 \mathrm{~h}$ to remove bubbles before spinning. After evacuation, the solution in the extruder barrel was put back into the extruder which was also heated to $80^{\circ} \mathrm{C}$. Fiber spinning started 5 min after the solution was transferred into the extruder. All solutions were injected through a $150 \mu \mathrm{m}$-diameter nozzle into the water bath. The air gap between the nozzle and the surface of the water bath was 3 $\mathrm{cm}$. We were unable to form continuous fibers with the $7.6 \mathrm{wt} \%$ cellulose/EMImDEP solution. Therefore, only cellulose fibers spun from 12.4, 15.2, and 18.0 wt \% cellulose/EMImDEP solutions are reported. The extrusion velocity $\left(V_{1}\right)$ was $0.4 \mathrm{~m} / \mathrm{s}$ while the haul off unit was continuously winding the coagulated fiber downstream at a winding velocity $\left(V_{2}\right)$ of $2.1 \mathrm{~m} / \mathrm{s}$ (draw ratio $=5.3$ ). After spinning, the fibers were immersed in tap water for 2 days to remove the ionic liquid solvent, with a change of water every $24 \mathrm{~h}$. Then the fibers were rolled and dried in a fume hood for a further $48 \mathrm{~h}$.

Characterization of Cellulose Fibers. The solvent was completely removed during processing, which was confirmed via FTIR in a previous study. ${ }^{15}$

The diameter measurements and the observations of the outer surfaces of cellulose fibers were carried out using a TM3030 Plus Tabletop scanning electron microscope produced by HITACHI (Berkshire, UK). Five filaments of each of the 12.4, 15.2, and 18.0 wt \% cellulose fibers were mounted onto a sample holder, without silver coating. Three scanning electron microscopy (SEM) images from three different locations of each fiber filament were obtained. From these images, three diameters were measured from three different locations along each filament using the ImageJ software package. Thus, 45 different locations' diameters were measured for $12.4,15.2$, and 18.0 wt \% cellulose fibers, respectively. Average SEM diameters and standard deviations were then determined (see Supporting Information Figure S4). The outer surfaces of 12.4, 15.2, and 18.0 wt \% cellulose fibers were also observed from these SEM micrographs.

To observe the cross sections of the fibers before and after tensile testing, SEM analysis was conducted. All fiber filaments were prepared using an Agar Scientific high-resolution sputter coater with $15 \mathrm{~nm}$ thick silver coating. The cross sections of fibers before tensile testing were obtained using liquid nitrogen. The cross-sectional areas of coated filaments were revealed (see Supporting Information Figure S5) and further observed using a JEOL IT300 SEM.

Wide angle X-ray diffraction (WAXD) patterns of single fiber filaments were obtained with an exposure time of $10 \mathrm{~h}$. A SAXSLAB GANESHA $300 \mathrm{XL}$ SAXS system in the School of Physics at University of Bristol was used for this study, consisting of an X-ray generator producing $\mathrm{Cu} \mathrm{K} \alpha$ radiation with a wavelength of $0.154 \mathrm{~nm}$, a sample stage and a detector inside a vacuum chamber, as well as data reduction and analysis software (SAXSGUI and IDL). The beam stop was $2 \mathrm{~mm}$, the beam size was $0.8 \mathrm{~mm}$, and the sample-to-detector 
distance was set at $100 \mathrm{~mm}$. Single fiber filaments were mounted straight and tight on a sample holder, which was located on the sample stage between the X-ray generator and detector.

Mechanical Properties of Cellulose Fibers. Three gauge lengths $(12,20$, and $30 \mathrm{~mm})$ of fibers were used for mechanical properties measurements to account for machine compliance and end effects (see Supporting Information Figure S6 and S7), according to ASTM standard C1557. Tensile testing was carried out using a DiaStron LEX820 single fiber tester (Hampshire, UK), containing a $20 \mathrm{~N}$ capacity load cell with a resolution of $0.5 \mathrm{mN}$. The tensile samples were prepared by mounting a single fiber filament between two plastic tabs. These tabs were located on a 20-slot linear plastic cassette with three different gauge lengths of 12, 20, and $30 \mathrm{~mm}$. Every single fiber filament was located straight and tightly on tabs using DYMAX 3193 UV adhesive (Wiesbaden, Germany). The tabs were clamped horizontally between a fixed jaw and a movable jaw on the fiber tester. All tensile samples were tested at the same strain rate of $10 \%$ / min. Tensile testing was controlled using the UvWin PC application. Tensile load and displacement data points were recorded automatically with an interval of $50 \mathrm{~ms}$ during the testing. Tensile strength and breaking strain were calculated from these data using eqs 1 and 2 , where $\sigma$ is the tensile stress, $\varepsilon$ is the tensile strain, $F$ is the tensile load, $d$ is the fiber diameter, $A$ is the fiber cross-sectional area, $l$ is the displacement, and $l_{0}$ is the initial gauge length.

Ten samples for each gauge length were prepared and tested to failure for 12.4, 15.2, and 18.0 wt \% cellulose fibers, respectively.

$$
\begin{aligned}
& \sigma=\frac{F}{A}=\frac{4 F}{\pi d^{2}} \\
& \varepsilon=\frac{l}{l_{0}}
\end{aligned}
$$

The goal of this work is to use the cellulose fibers as renewable and high stiffness reinforcement in composites. The effect of water absorption in cellulose fibers embedded in a polymer matrix is not going to be as high as for cellulose fibers used in clothing and textiles, where they will regularly be washed. Nevertheless, we believe water absorption can play an important effect even in cellulose fibers embedded in composites. To study the effects of water on the properties of cellulose fibers, 18.0 wt \% cellulose fibers were submerged in water for $24 \mathrm{~h}$. Ten wet 18.0 wt \% fiber filaments were mounted and glued on plastic tablets with a gauge length of 20 $\mathrm{mm}$ using UV adhesive, which took about $1 \mathrm{~h}$. After this preparation, the ten fiber filaments became almost dry. Their diameters were measured using a SEM method. The ten filament samples were tested to failure.

\section{RESULTS}

Anisotropy Study of Cellulose/EMImDEP Solutions. The cellulose/solvent solution films were observed with a polarized optical microscope. When the solutions are heated above the clearing temperature $\left(T_{\mathrm{c}}\right){ }^{16}$ the solution becomes isotropic, and the anisotropy pattern disappears. To study $T_{\mathcal{O}}$ 7.6, 12.4, 15.2, and 18.0 wt \% cellulose/EMImDEP solutions were heated from 25 to $90{ }^{\circ} \mathrm{C}$, while polarized optical micrographs were taken at different temperatures, respectively. The anisotropy of cellulose/EMImDEP solutions diminished gradually as the temperature increased and finally disappeared at $T_{\mathrm{c}}$.

When the concentration of cellulose was $7.6 \mathrm{wt} \%$, the solution was isotropic and appeared completely dark between crossed polarizers at 25 and $80{ }^{\circ} \mathrm{C}$ (Figure 2A). When the concentration of cellulose rose to $12.4 \mathrm{wt} \%$, a weakly nematic texture appeared at $25{ }^{\circ} \mathrm{C}$ and disappeared at $80{ }^{\circ} \mathrm{C}$ (Figure $2 \mathrm{~B})$. When the concentration of cellulose further increased to 15.2 wt \%, strong optical planar textures as a typical sign of anisotropy were observed at $25^{\circ} \mathrm{C}$ and also disappeared at 80
A
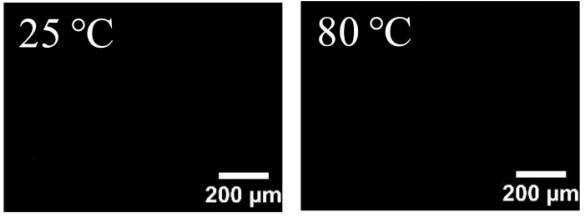

B
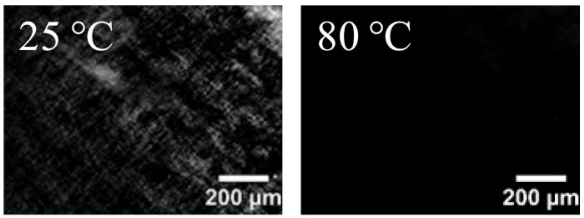

C
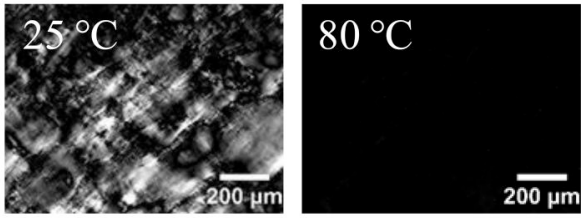

$\mathrm{D}$
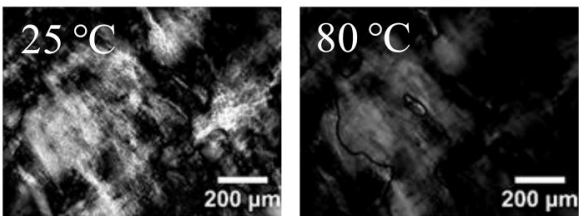

Figure 2. Typical polarized optical micrographs of (A) 7.6, (B) 12.4, (C) 15.2 , and (D) $18.0 \mathrm{wt} \%$ cellulose/EMImDEP solutions at 25 and $80{ }^{\circ} \mathrm{C}$.

${ }^{\circ} \mathrm{C}$ (Figure 2C). When the concentration of cellulose rose to $18.0 \mathrm{wt} \%$, the optical planar textures of cellulose/EMImDEP solutions became even stronger at $25{ }^{\circ} \mathrm{C}$ compared to $15.2 \mathrm{wt}$ $\%$ solutions, and the textures still existed after the solution was heated to $80{ }^{\circ} \mathrm{C}$ (Figure 2D). The $T_{\mathrm{c}}$ of $18.0 \mathrm{wt} \%$ cellulose/ EMImDEP solution was found to be between 85 and $90{ }^{\circ} \mathrm{C}$ (Figure 3). The $T_{\mathrm{c}}$ for 12.4 and $15.2 \mathrm{wt} \%$ solutions were found to be lower than 18 wt \% cellulose solution (see Supporting Information Figures S1 and S2).
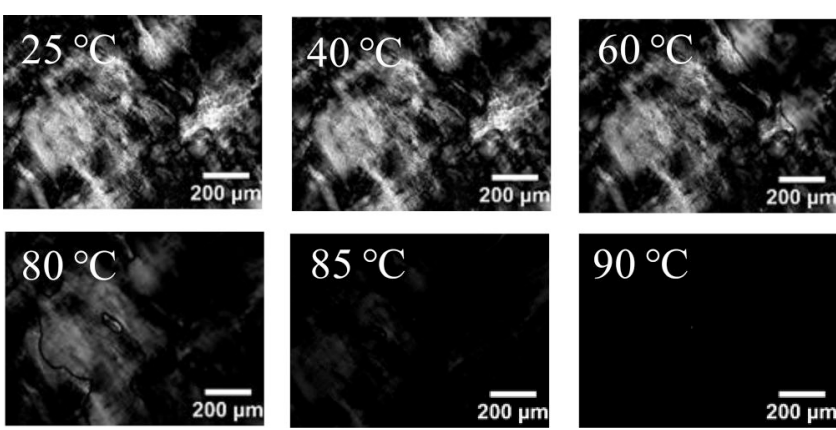

Figure 3. Typical anisotropy transition of $18.0 \mathrm{wt} \%$ cellulose/ EMImDEP solution heated from 25 to $90{ }^{\circ} \mathrm{C}$.

Diameter Measurements of Cellulose Fibers. To obtain a reliable measurement of fiber diameter we used two separate methods, namely SEM and optical microscopy of fiber cross sections embedded in the epoxy matrix.

SEM images of $12.4,15.2$, and 18.0 wt \% cellulose are shown in Figure 4A-I. Their average diameters as obtained from images are given in Table 1 . With the same draw ratio, no significant differences in the average diameters were observed for $12.4 \mathrm{wt} \%(22.0 \pm 1.4 \mu \mathrm{m}), 15.2 \mathrm{wt} \%(23.1 \pm 1.1 \mu \mathrm{m})$, and 

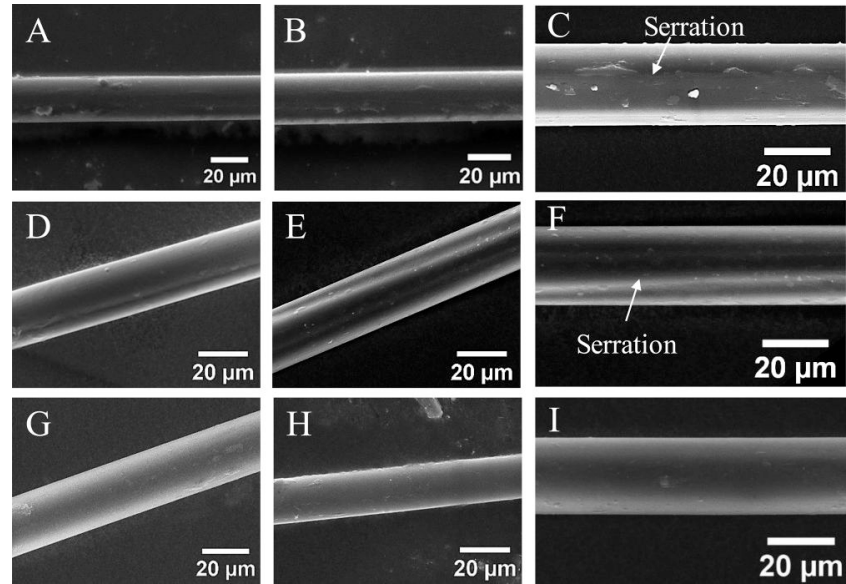

Figure 4. Typical SEM micrographs of (A-C) 12.4, (D-F) 15.2, and (G-I) 18.0 wt \% cellulose fibers for diameter measurement and outer surface observation.

18.0 wt $\%(20.8 \pm 3.0 \mu \mathrm{m})$ fibers (see Supporting Information Figures S4 and S5).

In the optical microscopy measurement method, the average cross-sectional area of 6-8 randomly selected filaments of 12.4 , 15.2, and $18.0 \mathrm{wt} \%$ cellulose fibers mounted in resin molds (see Figure S5 in the Supporting Information) were measured. The results of these cross section measurements were similar to the cross-sectional areas calculated using SEM diameters (Table 1). Hence we believe that our diameter measurements are accurate enough for this study.

Outer Surface Observation of Cellulose Fibers. SEM micrographs of the outer surfaces of cellulose fibers were taken (Figure 4C, F, and I). For 12.4 wt \% fibers, there were obvious serrations on the outer surface (Figure $4 \mathrm{C}$ ), as seen for viscose fibers. ${ }^{17}$ For a 15.2 wt \% fiber, the serrations seem to diminish in size (Figure 4F), but for the $18.0 \mathrm{wt} \%$ fiber, the serrations are not present at all and a smooth outer surface is observed (Figure 4I). It is known that the solution dope concentration affects the counter diffusion process between ionic liquid and water in the coagulation bath, which further influences the microscopic structures and mechanical properties of spun fibers. ${ }^{18}$ This might be the reason why the fiber outer surface became smoother and the serrations reduced as the cellulose concentration was increased to $18.0 \mathrm{wt} \%$. And the smoother outer surface of $18.0 \mathrm{wt} \%$ fiber also contributes to its better mechanical performance compared to 12.4 and 15.2 wt \% fibers. We have not done a comprehensive surface defect analysis of the fibers for $12.4 \%$, but it is likely that there are surface defects on fibers spun from 12 wt \% cellulose solution which may contribute to the reduction in the mechanical properties.

SEM Analyses for Fracturing Cross sections of Cellulose Fibers. The cross sections (perpendicular to the fiber axis) of the cellulose fibers before (Figure 5A-C) and
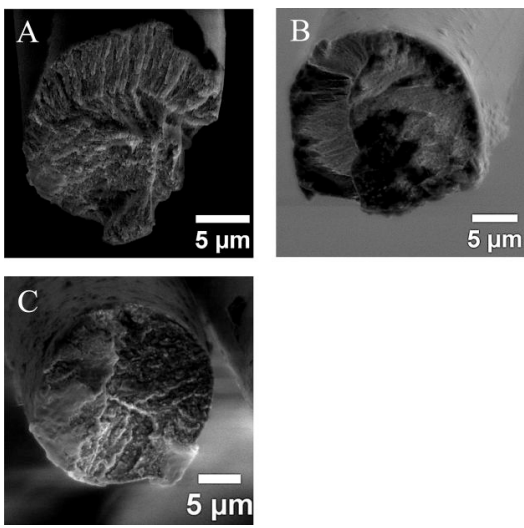

Figure 5. Typical SEM images of cross sections of (A) 12.4, (B) 15.2, and (C) $18.0 \mathrm{wt} \%$ cellulose fibers fractured using liquid nitrogen.

after (Figure 6A-F) tensile testing were observed using SEM. Before tensile testing, all cross-sectional shapes of the fibers
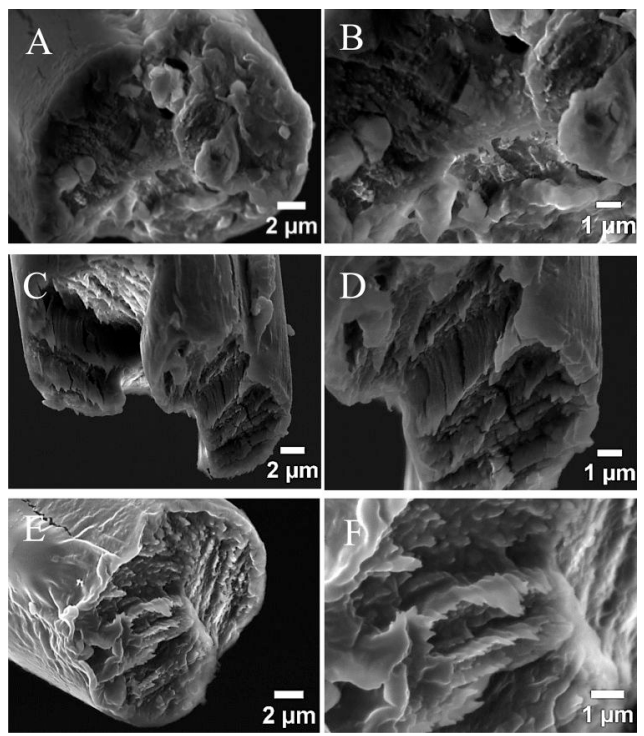

Figure 6. Typical SEM images of fractured surfaces of (A and B) 12.4, (C and D) 15.2, and (E and F) 18.0 wt \% cellulose fibers after tensile testing.

appear close to circular, similar to NMMO-type fibers, and different to the serrated shape of viscose fibers. All fibers seem to have the uniform structure throughout the cross-section without any visible large size voids. After tensile testing, the cross sections of cellulose fibers remain circular without obvious necking behavior (Figure 6). As demonstrated, the cellulose fibers are more apt to lateral slitting like NMMO fibers instead of ductile fracture like viscose fibers, ${ }^{19}$ as the increase of cellulose concentration (Figure 6).

Table 1. Average SEM Diameter, Average Optical Microscopy Diameter Calculated from Resin Cross-Sectional Area, SEM Cross-Sectional Area, and Resin Cross-Sectional Area of 12.4, 15.2, and 18.0 wt \% Cellulose Fibers

material

12.4 wt $\%$ cellulose fibers

15.2 wt \% cellulose fibers

18.0 wt \% cellulose fibers
SEM diameter $(\mu \mathrm{m})$

$$
\begin{aligned}
& 22.0( \pm 1.4) \\
& 23.1( \pm 1.1) \\
& 20.8( \pm 3.0)
\end{aligned}
$$

optical microscopy diameter $(\mu \mathrm{m})$

$$
\begin{aligned}
& 22.2( \pm 0.4) \\
& 24.7( \pm 0.7) \\
& 20.2( \pm 0.8)
\end{aligned}
$$

SEM cross-sectional area $\left(\mu \mathrm{m}^{2}\right)$

$$
\begin{aligned}
& 380.4( \pm 20.1) \\
& 419.2( \pm 36.6) \\
& 348.1( \pm 100.2)
\end{aligned}
$$

resin cross-sectional area $\left(\mu \mathrm{m}^{2}\right)$

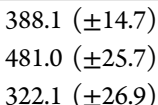


Wide Angle X-ray Diffraction of Cellulose Fibers. Figure $7 \mathrm{~A}-\mathrm{C}$ shows the two-dimensional WAXD diffraction
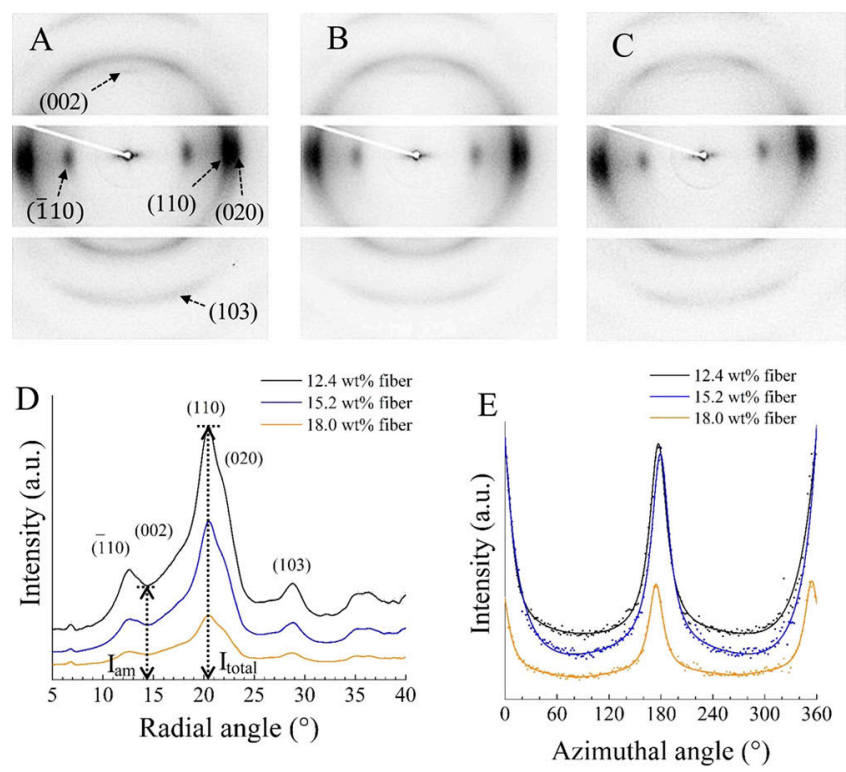

Figure 7. Typical WAXD patterns of (A) 12.4, (B) 15.2, and (C) 18.0 wt \% cellulose fibers, as well as their (D) radial scanning data and (E) azimuthal scanning data from the (110) peak.

patterns for single cellulose fibers. A narrow strip of intensity on each WAXD pattern was taken for the regrouping, which was much less than the radial width of all peaks. Radial scanning data (intensity against $2 \theta$ ) were obtained and are reported in Figure 7D. The Bragg peaks shown only occur for a cellulose II structure, a widely known crystal structure of regenerated cellulose after dissolution. ${ }^{8,20}$

Two Bragg peaks appear at $20.6^{\circ}$ and $21.6^{\circ}$ corresponding to the reflection planes (110) and (020) respectively, and a small peak at $28.8^{\circ}$ corresponding to the (103) plane. A secondary peak appears at $12.7^{\circ}$ corresponding to the cellulose (110) plane, indicating a structural transformation into cellulose II (Figure 7B). The shift of the main (110) peak from $22.5^{\circ}$ of cellulose I to $20.6^{\circ}$ of regenerated cellulose is evidence for a nonrecoverable change in the cellulose lattice structure after regeneration due to the diffused IL. ${ }^{21}$
For regenerated cellulose, cellulose II is the most common structure, possessing an ideal monoclinic $P 2_{1}$ structure with unit parameters of $a=0.801 \mathrm{~nm}, b=0.904 \mathrm{~nm}, c=1.036 \mathrm{~nm}, \alpha$ $=\beta=90^{\circ}$, and $\gamma=117.1^{\circ}$. ${ }^{22}$ Unit cell dimensions were calculated for our fibers using the measured q-spaces of the (110), (110), (002), (020), and (103) planes from the WAXD patterns. The measured unit parameters of cellulose in our fibers were found to be $a=0.78 \mathrm{~nm}, b=0.90 \mathrm{~nm}, c=1.03 \mathrm{~nm}$, and $\gamma=117.2^{\circ}$ for the $12.4 \mathrm{wt} \%$ fiber, $a=0.78 \mathrm{~nm}, b=0.90$ $\mathrm{nm}, c=1.03 \mathrm{~nm}$, and $\gamma=116.9^{\circ}$ for the $15.2 \mathrm{wt} \%$ fiber, and $a=$ $0.78 \mathrm{~nm}, b=0.90 \mathrm{~nm}, c=1.03 \mathrm{~nm}$, and $\gamma=116.0^{\circ}$ for the 18.0 wt \% fiber. Our values appear to differ somewhat to those published in the literature (particularly for the $a$-axis) which may be due to a large number of diffraction intensities overlapping each other in the X-ray data for the cellulose II structure. $^{23}$

The fraction of crystalline material in cellulose fibers, or crystallinity, can be estimated using the crystallinity index (CrI) developed by $\mathrm{Segal}^{24}$ in eq 3, for the comparison of similar structure materials prepared by similar methods.

$$
\operatorname{CrI}(\%)=\frac{I_{\text {total }}-I_{\mathrm{am}}}{I_{\text {total }}} \times 100
$$

where $I_{\text {total }}$ is the total scattered intensity at the main peak, and the $I_{a m}$ is the minimum scattered intensity between the main and secondary peaks for cellulose. ${ }^{25}$ To use CrI, an assumption that there is only a single crystalline phase along with an amorphous phase has been applied. ${ }^{26}$ For cellulose II structure in regenerated cellulose fibers in our work, the main peak appears as a doublet composed of (110) and (002) peaks at $20.6^{\circ}$ and $21.6^{\circ}$, and the secondary peak appears at $12.7^{\circ}$ corresponding to $(\overline{1} 10)$ plane $^{27,28}$ (Figure $\left.7 \mathrm{D}\right)$. Therefore, the CrI of 12.4, 15.2, and 18.0 wt \% cellulose fibers are calculated using $I_{110}$ and $I_{\mathrm{am}}$ (Figure 7D) after subtraction of the background signal measured without cellulose, which are $65.0 \%, 66.8 \%$, and $64.4 \%$ correspondingly (Table 2 ). It appears that the cellulose fibers with different cellulose concentrations in this work have similar crystallinities $(\sim 65 \%)$ when produced under the same condition. The CrI of cellulose in our fibers are similar to the cellulose fibers regenerated by Rehatekar et al. ${ }^{8}$

In a stretched fiber, the cellulose chains have a preferred orientation with their longitudinal axes parallel to the deformation direction, which appear as concentrated intensity as two arcs in the diffraction ring, in the azimuthal direction

Table 2. Degree of Polymerization (DP), Average SEM Diameter, Crystallinity Index (CrI), Full Width at Half Maximum (FWHM), Orientation Function $(f)$, Magnitude of the Orientation Parameter $\left\langle\sin ^{2} \Delta \phi\right\rangle$ Data of the ( 110$)$ Azimuthal Peaks, Best Young's Modulus, and Corresponding Tensile Strength of 12.4, 15.2, and 18.0 wt \% Cellulose Fibers, Compared to Regenerated Cellulose Fibers Reported by Previous Researchers

\begin{tabular}{|c|c|c|c|c|c|c|c|c|}
\hline material & DP & diameter $(\mu \mathrm{m})$ & $\begin{array}{l}\text { Young's modulus } \\
\text { (GPa) }\end{array}$ & $\begin{array}{l}\text { tensile strength } \\
(\mathrm{MPa})\end{array}$ & $\begin{array}{l}\mathrm{CrI} \\
(\%)\end{array}$ & $\begin{array}{c}\text { FWHM of ( }(\overline{1} 10) \\
\text { peak (deg) }\end{array}$ & $\begin{array}{c}f \text { of }(\overline{1} 10) \\
\text { peak }\end{array}$ & $\begin{array}{c}\left\langle\sin ^{2} \Delta \phi\right\rangle(\overline{1} 10) \\
\text { of peak }\end{array}$ \\
\hline $\begin{array}{l}12.4 \text { wt \% } \\
\text { cellulose fibers }\end{array}$ & $200-220$ & $22.0( \pm 1.4)$ & $14.8( \pm 2.3)$ & $215.5( \pm 11.9)$ & 65.0 & 24 & 0.77 & 0.08 \\
\hline $\begin{array}{l}15.2 \text { wt \% } \\
\text { cellulose fibers }\end{array}$ & $200-220$ & $23.1( \pm 1.1)$ & $15.7( \pm 1.6)$ & $226.4( \pm 10.0)$ & 66.8 & 25 & 0.76 & 0.08 \\
\hline $\begin{array}{l}18.0 \mathrm{wt} \% \\
\text { cellulose fibers }\end{array}$ & $200-220$ & $20.8( \pm 3.0)$ & $22.4( \pm 1.4)$ & $304.7( \pm 12.7)$ & 64.4 & 21 & 0.80 & 0.07 \\
\hline Luo et al. ${ }^{9}$ & $200-220$ & $300-400$ & & $73.8( \pm 2.2)$ & & & & \\
\hline Lim et al. $^{42}$ & & $5-10$ & $11-13$ & $280-400$ & & & & \\
\hline He et al. $^{36}$ & 650 & & 5.1 & $204.0( \pm 6.0)$ & & & 0.71 & \\
\hline Rahatekar et al. ${ }^{8}$ & 820 & $23.2( \pm 1.8)$ & $13.1( \pm 1.1)$ & $198.0( \pm 25.0)$ & 62 & & & \\
\hline Sixta et al. ${ }^{37}$ & $1026-1133$ & & $23.4( \pm 3.5)$ & $\sim 694.0$ & & & 0.73 & \\
\hline
\end{tabular}


(Figure 7A and $\mathrm{B}$ ). The intensities of the diffraction rings corresponding to the cellulose $(\overline{1} 10)$ planes were plotted as a function of azimuthal angle and fitted using a Lorentzian function (Figure 7E). The full width at half-maximum (fwhm) is the difference in the angle across the peak where the intensity is $50 \%$ of the maximum value. ${ }^{29}$ The fwhm of cellulose peaks corresponding to the ( 110$)$ planes were calculated as an indication of the degree of alignment of cellulose chains in all fibers, and found to be $24^{\circ}, 25^{\circ}, 21^{\circ}$, respectively (Table 2 ). The lower the value of fwhm, the higher is the degree of alignment of the cellulose chains. ${ }^{30}$ The regenerated cellulose fibers appear to possess aligned cellulose chains, as exhibited by the sharp azimuthal peaks in Figure 7E.

The "orientation parameter", $f$, first proposed by Hermans, ${ }^{31}$ can be used to characterize the extent of cellulose crystallite orientation in our fibers. This parameter is defined as the mean coefficient of the second-order Legendre polynomial, $P_{2}(\cos \theta)$, where $\theta$ is the polar disorientation angle of a crystallite relative to the fiber axis and the angle brackets indicate an average over all crystallites (eq 4). ${ }^{32}$ The cellulose crystallites would have a perfect orientation perpendicular to the fiber axis when $f=$ -0.5 , and a perfect orientation parallel with fiber axis when $f=$ 1.0. For a uniaxial fiber, $f$ may be measured directly from the azimuthal intensity distribution, $\rho(\phi)$, of a paratropic peak (i.e., one resulting from planes parallel to the crystallite axis) in the X-ray diffraction pattern (eq 5).

$$
\begin{aligned}
& f=\left\langle P_{2}(\cos \theta)\right\rangle=\frac{\left(3\left\langle\cos ^{2} \theta\right\rangle-1\right)}{2} \\
& f=\left\langle P_{2}(\cos \theta)\right\rangle=(-2) \frac{\int_{0}^{\pi} \rho(\phi) P_{2}(\cos \phi) \sin \phi \mathrm{d} \phi}{\int_{0}^{\pi} \rho(\phi) \sin \phi \mathrm{d} \phi}
\end{aligned}
$$

where the factor of -2 arises because the center of the peak is at right angles to the fiber axis ${ }^{33}$ and only tilts of the crystallites in the plane containing the $\mathrm{X}$-ray beam and the fiber axis broaden the diffraction peak.

Another commonly used measure ${ }^{34}$ of the orientational order is $\left\langle\sin ^{2} \theta\right\rangle$ which is closely related to $f$ (eq 6 ), where the angle $\theta$ is the crystallite angle. For a uniaxial fiber, this can be measured directly from the azimuthal intensity distribution, $\rho(\phi)$, of a diatropic peak (i.e., one resulting from planes perpendicular to the crystallite axis) by eq 7 .

$$
\begin{aligned}
& \left\langle\sin ^{2} \theta\right\rangle=\frac{2}{3}(1-f) \\
& \left\langle\sin ^{2} \theta\right\rangle=\frac{\int_{0}^{\pi} \rho(\phi) \sin ^{3} \phi \mathrm{d} \phi}{\int_{0}^{\pi} \rho(\phi) \sin \phi \mathrm{d} \phi}
\end{aligned}
$$

However, for a paratropic peak (i.e., one resulting from planes parallel to the crystallite axis) from a uniaxial fiber the equation becomes

$$
\left\langle\sin ^{2} \theta\right\rangle=2\left\langle\sin ^{2} \Delta \phi\right\rangle=2 \frac{\int_{0}^{\pi} \rho(\phi) \cos ^{2} \phi \sin \phi \mathrm{d} \phi}{\int_{0}^{\pi} \rho(\phi) \sin \phi \mathrm{d} \phi}
$$

where, again, the factor of 2 arises because only tilts of the crystallites in the plane broaden the diffraction peak and $\Delta \phi$ is measured from $\phi=\pi / 2$. In the literature, the spread of a paratropic peak, $\left\langle\sin ^{2} \Delta \phi\right\rangle$, rather than the spread of the crystallites, $\left\langle\sin ^{2} \theta\right\rangle$, is often evaluated and used as an experimental observable to gauge the quality of orientation..$^{35}$ It can be calculated directly from $f$ (eq 9).

$$
\left\langle\sin ^{2} \Delta \phi\right\rangle=\frac{(1-f)}{3}
$$

The orientation parameter values for the cellulose fibers were calculated from the intensity distribution in a ring containing the $(\overline{1} 10)$ peak, with background estimated from adjacent rings and subtracted using IDL. The value of $f$ was calculated from eq 5. The highest orientation function $(f)$ value was observed for 18 wt \% fibers $(0.80)$ as shown in Table 2 , indicating the very good orientation of the cellulose chains along the fiber axis compared to the fibers produced previously by the groups of $\mathrm{He}^{36}(f=0.71)$ and $\operatorname{Sixta}^{37}(f=0.73)$.

For comparison with literature values, $\left\langle\sin ^{2} \Delta \phi\right\rangle$ was also calculated using eq 9, and the results are given in Table 2. This parameter has been previously utilized to determine the crystalline orientation parameter of PBO (poly $p$-phenylene benzobisoxazole) fibers. ${ }^{38}$ Crystalline orientation is inversely proportional to the value of the orientation parameter, and therefore, for a perfect orientation, $\left\langle\sin ^{2} \Delta \phi\right\rangle$ is equal to zero. The $\left\langle\sin ^{2} \Delta \phi\right\rangle$ values for our fibers are shown in Table 2 . The lowest orientation parameter $\left(\left\langle\sin ^{2} \Delta \phi\right\rangle=0.07\right)$ i.e. highest orientation of crystallites along the fiber axis was observed for the $18 \mathrm{wt} \%$ fibers. The orientation parameter of our $18 \mathrm{wt} \%$ fibers (0.07) indicates lower orientational order than the cellulose fibers $\left(\left\langle\sin ^{2} \Delta \phi\right\rangle=\sim 0.01\right)$ regenerated from a liquid crystalline cellulose solution reported in the literature. ${ }^{39}$ Lyocell cellulose fibers have also been reported to possess a lower orientation parameter of 0.05 at the center of the fiber, indicating a higher crystallite orientation along the fiber axis. ${ }^{40}$

Tensile Testing of Cellulose Fibers. Typical tensile stress-strain curves for 12.4, 15.2, and $18.0 \mathrm{wt} \%$ cellulose fibers with a gauge length of $20 \mathrm{~mm}$ are shown in Figure 8. It is

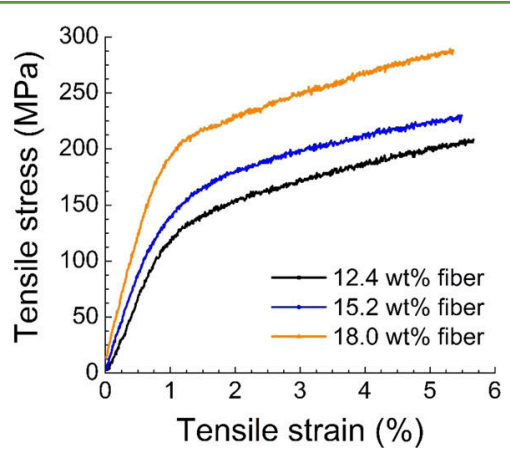

Figure 8. Typical tensile stress-strain curves for 12.4, 15.2, and 18.0 wt $\%$ regenerated cellulose fibers.

found that, as the cellulose concentration increased from 12.4 to $15.2 \mathrm{wt} \%$, the mechanical properties (Young's modulus, tensile strength, and breaking strain) of cellulose fibers did not show any obvious differences. As the cellulose concentration further increased to $18.0 \mathrm{wt} \%$, both Young's modulus and tensile strength of cellulose fibers significantly increased (Figure 8 and Table 3 ), while the breaking strain remained similar.

With the same increment in cellulose concentration (2.8 wt $\%)$, the increase in Young's modulus was significantly different for 15.2 and 18.0 wt \% cellulose fibers. When the concentration of cellulose increased from 12.4 to $15.2 \mathrm{wt} \%$, Young's modulus only increased slightly from 15.8 to $16.2 \mathrm{GPa}$. When the concentration further increased to $18.0 \mathrm{wt} \%$, Young's modulus 
Table 3. Young's Modulus, Tensile Strength, and Breaking Strain of 12.4, 15.2, and 18.0 wt \% Cellulose Fibers with Different Gauge Lengths of 12, 20, and $30 \mathrm{~mm}$

\begin{tabular}{ccccc}
\hline material & $\begin{array}{c}\text { gauge } \\
\text { length } \\
(\mathrm{mm})\end{array}$ & $\begin{array}{c}\text { Young's } \\
\text { modulus } \\
(\mathrm{GPa})\end{array}$ & $\begin{array}{c}\text { tensile strength } \\
(\mathrm{MPa})\end{array}$ & $\begin{array}{c}\text { breaking } \\
\text { strain }(\%)\end{array}$ \\
$\begin{array}{c}12.4 \text { wt \% } \\
\text { cellulose }\end{array}$ & 12 & $13.5( \pm 1.5)$ & $207.3( \pm 13.6)$ & $5.0( \pm 0.2)$ \\
fibers & 20 & $14.4( \pm 0.5)$ & $212.2( \pm 16.6)$ & $5.5( \pm 1.2)$ \\
15.2 wt \% & 30 & $14.8( \pm 2.3)$ & $215.5( \pm 11.9)$ & $5.0( \pm 0.1)$ \\
cellulose & 12 & $15.0( \pm 2.3)$ & $232.4( \pm 4.6)$ & $4.9( \pm 0.1)$ \\
fibers & 20 & $15.7( \pm 1.6)$ & $226.4( \pm 10.0)$ & $5.7( \pm 1.1)$ \\
18.0 wt \% & 30 & $14.7( \pm 4.6)$ & $226.0( \pm 6.4)$ & $4.7( \pm 0.4)$ \\
cellulose & 12 & $19.3( \pm 3.1)$ & $290.0( \pm 36.3)$ & $5.6( \pm 0.7)$ \\
fibers & 20 & $22.4( \pm 1.4)$ & $304.7( \pm 12.7)$ & $6.5( \pm 0.7)$ \\
& 30 & $18.9( \pm 3.2)$ & $252.3( \pm 35.2)$ & $5.2( \pm 0.8)$ \\
\hline
\end{tabular}

rose from 16.2 to $22.8 \mathrm{GPa}$. As cellulose concentration increased from 12.4 to $18.0 \mathrm{wt} \%$, the tensile strength of cellulose fibers increased from 207.3-215.5 to 252.3-304.7 $\mathrm{MPa}$. Meanwhile, the breaking strain for fiber samples with different gauge length exhibited no change (Table 3 ).

After wetting in water for $24 \mathrm{~h}$, the swelling of cellulose fibers was determined by the increment of fiber diameter from 20.8 to $25.4 \mu \mathrm{m}$. Meanwhile, a moderate reduction on the mechanical properties of cellulose fibers (5.9 GPa in Young's modulus and $67 \mathrm{MPa}$ in tensile strength) was also observed.

\section{DISCUSSION}

In this study, the cellulose/EMImDEP solution appeared isotropic for a cellulose concentration of $7.6 \mathrm{wt} \%$, weakly nematic for a concentration of 12.4 wt $\%$ and anisotropic at 15.2 and $18.0 \mathrm{wt} \%$. Song et al. studied the anisotropic behaviors of $\mathrm{MCC} / \mathrm{AMImCl}^{10}$ and $\mathrm{MCC} / \mathrm{EMImAc}^{14}$ solutions. Their solutions possessed similar isotropic forms at lower concentrations (at $7 \mathrm{wt} \%$ ), at lower threshold concentrations (9-10 wt \%) appeared to show a weak optical texture and at higher concentrations (11-18 wt \%) gave a strong optical texture. Boerstoel et al. reported that the $T_{\mathrm{c}}$ of cellulose solutions increases as the concentration of polymer increases, ${ }^{16}$ which is named lyotropic behavior. ${ }^{41}$ The anisotropic appearance may be attributed to an increase in the alignment of cellulose chains during dissolution and the resistance for the migration into a random state; this is thought to be due to the high shear viscosity of the cellulose/EMImDEP solution and the large number density of cellulose. ${ }^{3,41}$ When the concentration of cellulose in our study was $7.6 \mathrm{wt} \%$, the $T_{\mathrm{c}}$ of cellulose/EMImDEP solution was lower than room temperature which is hard to observe under a polarized optical microscope. When the concentration of cellulose is $12.4 \mathrm{wt} \%$ or higher, the $T_{\mathrm{c}}$ of cellulose/EMImDEP solution increased above room temperature, and the nematic texture of solution appears under polarized light. Upon heating, the shear viscosity of the anisotropic solution decreased while the cellulose molecules became disordered. Finally, the solution reached an isotropic state at $T_{c}$. This inversely proportional anisotropytemperature performance is named thermotropic behavior. Under the collective effect of thermotropic and lyotropic behaviors, the anisotropy of the cellulose solutions diminished gradually as temperature increased and finally disappeared at 80 ${ }^{\circ} \mathrm{C}$ for 12.4 and 15.2 wt \% solutions, while the anisotropy disappeared at an increasing temperature of $85<T_{\mathrm{c}}<90{ }^{\circ} \mathrm{C}$ for 18.0 wt $\%$ solution.
The $T_{c}$ of 11.4 wt \% cellulose/phosphoric acid solution obtained by Boerstoel et al. was as low as $45{ }^{\circ} \mathrm{C}$. ${ }^{16}$ Song and his group conducted similar observations of anisotropic transitions on 16 wt \% MCC/AMImCl solutions with a similar range of 75 $<T_{\mathrm{c}}<80^{\circ} \mathrm{C}$, ${ }^{10}$ as well as on $14 \mathrm{wt} \% \mathrm{MCC} / \mathrm{EMImAc}$ solution with a range of $75<T_{\mathrm{c}}<85{ }^{\circ} \mathrm{C}$. Therefore, the $18.0 \mathrm{wt} \%$ cellulose/EMImDEP solution prepared in this study possessed a higher $T_{c}$ than most previous studies, indicating better selfaccessibility of cellulose chains dispersed in EMImDEP.

The difference in $T_{\mathrm{c}}$ of $12.4,15.2$, and $18.0 \mathrm{wt} \%$ cellulose/ EMImDEP solutions also indicates that during the fiber spinning process at $80{ }^{\circ} \mathrm{C}$, the 12.4 and $15.2 \mathrm{wt} \%$ fibers were produced from isotropic solutions, while $18.0 \mathrm{wt} \%$ fibers were produced from an anisotropic solution. This could be the reason why the mechanical properties of $18.0 \mathrm{wt} \%$ cellulose fibers were significantly higher than those of 12.4 and 15.2 wt \% cellulose fibers.

The fibers reported in this study, which was regenerated using low molecular weight $(\sim 220)$ cellulose, showed moderately high Young's modulus $(\sim 22 \mathrm{GPa})$ and tensile strength $(\sim 305 \mathrm{MPa})$. We have compared these values with other natural polymer fibers and fiber-based materials (balsa wood, wool, bone, worm silk and spider silk, etc.) and currently used commercial viscose (Figure 9). The average Young's

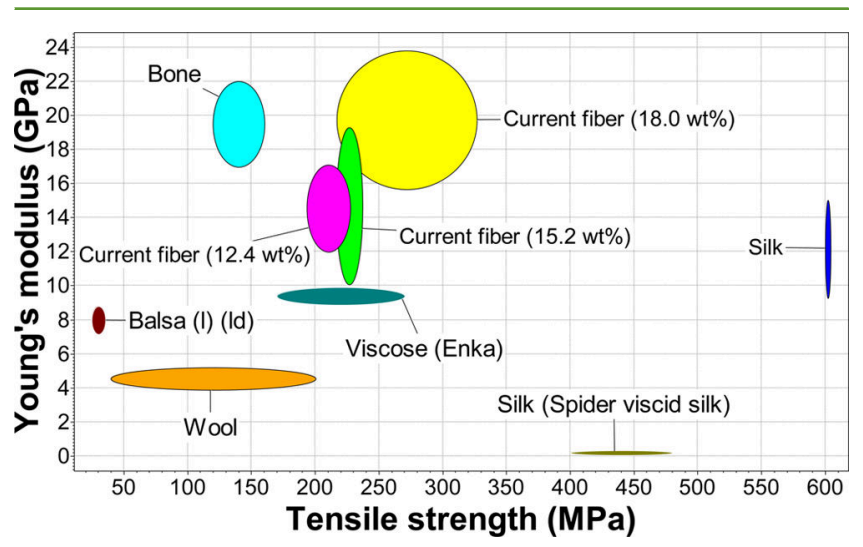

Figure 9. Mechanical properties of $12.4,15.2$, and 18.0 wt \% regenerated cellulose fibers, compared to natural polymer fibers (balsa, wool, bone, worm silk, and spider silk) as well as commercial viscose (Enka) cellulose fibers. ${ }^{43}$

modulus (for $18.0 \mathrm{wt} \%$ fibers) is higher than all these fibers; however, the tensile strength is lower than silk fibers from silkworms and spiders. Our cellulose fibers also showed good mechanical performance compared with regenerated cellulose fibers from higher DP cellulose using ionic liquids as solvents reported by some of the previous researchers (Table 2). The regenerated cellulose fibers from $\mathrm{MCC} / \mathrm{AMImCl}$ solution reported by Luo et al. had a much lower tensile strength of $73.8 \mathrm{MPa}{ }^{9}$ Lim et al. wet-spun cellulose fibers from a rice straw cellulose/NMMO solution, with a much lower Young's modulus in the range 11.0-13.0 GPa, but tensile strength similar to our fibers $(280.0-400.0 \mathrm{MPa}) .{ }^{42} \mathrm{He}$ et al. dry-jet wet spun cellulose fibers from a 14.5 wt \% cellulose/AMImCl solution (cellulose DP $=650$ ) with Young's moduli as low as 5.1 GPa and a lower strength of $204.0 \mathrm{MPa} .{ }^{36}$ Rahatekar et al. dry-jet wet spun cellulose fibers using EMImAc from a smaller spinneret $(120 \mu \mathrm{m})$ at a draw ratio of 1.5 , with a finer diameter of $23.2 \mu \mathrm{m}$, yielding fibers with a Young's modulus of $13.1 \mathrm{GPa}$ and strength of $198.0 \mathrm{MPa}^{8}$ Sixta et al. have recently 
manufactured cellulosic fibers (Ioncell-F) with a very good Young's modulus (23.4 GPa) and tensile strength ( 694.0 $\mathrm{MPa}){ }^{37}$ However, they used a high DP cellulose (DP 1095) and their spinning procedure used a different $\mathrm{IL}$ as a solvent, without forming an anisotropic solution before fiber spinning. The manufacturing method developed in this study will be very useful to convert low molecular weight waste cellulose, which is normally used for medical tablets, food-ingredient, etc., to fibers with good mechanical properties which could be used in the composite engineering industry.

\section{CONCLUSIONS}

We have developed a novel manufacturing method for stiff regenerated cellulose fibers spun from an anisotropic solution of low molecular weight cellulose dissolved in EMImDEP. The 18.0 wt \% cellulose/EMImDEP solution appeared highly anisotropic with a strong sign of anisotropy and clearing temperature $\left(T_{\mathrm{c}}\right)$ between $85<T_{\mathrm{c}}<90{ }^{\circ} \mathrm{C}$. Scanning electron microscopy graphs demonstrated that the cellulose fibers possessed circular, dense and homogeneous cross sections, without any visible voids. The wide angle $\mathrm{X}$-ray diffraction and mechanical testing of fibers spun from 12.4, 15.2, and $18.0 \mathrm{wt} \%$ cellulose solution confirmed that 18.0 wt \% cellulose fiber possessed the highest molecular alignment and therefore mechanical properties (Young's modulus $\sim 22 \mathrm{GPa}$; tensile strength $\sim 305 \mathrm{MPa})$. Despite using a low molecular weight cellulose, we were able to achieve superior tensile modulus from our fibers compared to previous studies which used higher molecular weights. These findings open up a potential route to convert low-performance cellulose waste into high-performance engineering fibers.

\section{ASSOCIATED CONTENT}

\section{S Supporting Information}

The Supporting Information is available free of charge on the ACS Publications website at DOI: 10.1021/acssuschemeng.6b00555.

Clearing temperature of $12.4,15.2$, and 18.0 wt \% cellulose/EMImDEP solutions, Figures S1-S3; diameter measurements, cross-section observation, and machine compliance (Cs) study of $12.4,15.2$, and $18.0 \mathrm{wt} \%$ cellulose fibers, Equations S1-S3, and Figures S4-S7 (PDF)

\section{AUTHOR INFORMATION}

\section{Corresponding Authors}

*Telephone: +44 (0) 139272 5515. Fax: +44(0) 139221 7965. E-mail: s.j.eichhorn@exeter.ac.uk (S.J.E.).

*Telephone: +44 (0)1173315330. Fax: +44(0)1173315360. Email: Sameer.Rahatekar@bristol.ac.uk (S.S.R.).

\section{Funding}

This research work is funded by Engineering and Physical Science Research Council (EPSRC, grant code EP/L017679/ 1).

\section{Notes}

The authors declare no competing financial interest.

\section{ACKNOWLEDGMENTS}

We would like to acknowledge funding from the Engineering and Physical Science Research Council (EPSRC, grant code EP/L017679/1). We thank Jon Jones for help with SEM studies carried out in the Chemical Imaging Facility, University of Bristol. The SEM and the Ganesha X-ray scattering apparatus used for this work were supported by an EPSRC Grant "Atoms to Applications" Grant ref EP/K035746/1.

\section{REFERENCES}

(1) Pinkert, A.; Marsh, K. N.; Pang, S.; Staiger, M. P. Ionic liquids and their interaction with cellulose. Chem. Rev. 2009, 109 (12), 67126728.

(2) Moon, R. J.; Martini, A.; Nairn, J.; Simonsen, J.; Youngblood, J. Cellulose nanomaterials review: structure, properties and nanocomposites. Chem. Soc. Rev. 2011, 40 (7), 3941-3994.

(3) Swatloski, R. P.; Spear, S. K.; Holbrey, J. D.; Rogers, R. D. Dissolution of cellose with ionic liquids. J. Am. Chem. Soc. 2002, 124 (18), 4974-4975.

(4) Alqus, R.; Eichhorn, S. J.; Bryce, R. A. Molecular Dynamics of Cellulose Amphiphilicity at the Graphene-Water Interface. Biomacromolecules 2015, 16 (6), 1771-1783.

(5) Hermanutz, F.; Gähr, F.; Uerdingen, E.; Meister, F.; Kosan, B. New Developments in Dissolving and Processing of Cellulose in Ionic Liquids. Macromol. Symp. 2008, 262 (1), 23-27.

(6) Zhu, S. D.; Wu, Y. X.; Chen, Q. M.; Yu, Z. N.; Wang, C. W.; Jin, S. W.; Ding, Y. G.; Wu, G. Dissolution of cellulose with ionic liquids and its application: a mini-review. Green Chem. 2006, 8 (4), 325-327.

(7) Fox, D. M.; Awad, W. H.; Gilman, J. W.; Maupin, P. H.; De Long, H. C.; Trulove, P. C. Flammability, thermal stability, and phase change characteristics of several trialkylimidazolium salts. Green Chem. 2003, 5 (6), 724-727.

(8) Rahatekar, S. S.; Rasheed, A.; Jain, R.; Zammarano, M.; Koziol, K. K.; Windle, A. H.; Gilman, J. W.; Kumar, S. Solution spinning of cellulose carbon nanotube composites using room temperature ionic liquids. Polymer 2009, 50 (19), 4577-4583.

(9) Luo, Z. Q.; Wang, A. Q.; Wang, C. Z.; Qin, W. C.; Zhao, N. N.; Song, H. Z.; Gao, J. G. Liquid crystalline phase behavior and fiber spinning of cellulose/ionic liquid/halloysite nanotubes dispersions. J. Mater. Chem. A 2014, 2 (20), 7327-7336.

(10) Song, H. Z.; Zhang, J.; Niu, Y. H.; Wang, Z. G. Phase transition and rheological behaviors of concentrated cellulose/ionic liquid solutions. J. Phys. Chem. B 2010, 114 (18), 6006-6013.

(11) Dave, V.; Glasser, W. G.; Wilkes, G. L. Cellulose-based fibers from liquid-crystalline solutions. 2. Processing and morphology of acetate butyrate esters. J. Polym. Sci., Part B: Polym. Phys. 1993, 31 (9), $1145-1161$.

(12) Postema, A. R.; Liou, K.; Wudl, F.; Smith, P. Highly oriented, low-modulus materials from liquid-crystalline polymers - The ultimate penalty for solubilizing alkyl side-chains. Macromolecules 1990, 23 (6), $1842-1845$

(13) Northolt, M. G.; Boerstoel, H.; Maatman, H.; Huisman, R.; Veurink, J.; Elzerman, H. The structure and properties of cellulose fibres spun from an anisotropic phosphoric acid solution. Polymer 2001, 42 (19), 8249-8264.

(14) Song, H.; Niu, Y.; Wang, Z.; Zhang, J. Liquid crystalline phase and gel-sol transitions for concentrated microcrystalline cellulose (MCC)/1-ethyl-3-methylimidazolium acetate (EMIMAc) solutions. Biomacromolecules 2011, 12 (4), 1087-1096.

(15) Zhu, C.; Chen, J.; Koziol, K. K.; Gilman, J. W.; Trulove, P. C.; Rahatekar, S. S. Effect of fibre spinning conditions on the electrical properties of cellulose and carbon nanotube composite fibres spun using ionic liquid as a benign solvent. eXPRESS Polym. Lett. 2014, 8 (3), 154-163.

(16) Boerstoel, H.; Maatman, H.; Westerink, J. B.; Koenders, B. M. Liquid crystalline solutions of cellulose in phosphoric acid. Polymer 2001, 42 (17), 7371-7379.

(17) Muller, M.; Riekel, C.; Vuong, R.; Chanzy, H. Skin/core microstructure in viscose rayon fibres analysed by X-ray microbeam and electron diffraction mapping. Polymer 2000, 41 (7), 2627-2632. 
(18) Hou, C.; Qu, R. J.; Liang, Y.; Wang, C. G. Kinetics of diffusion in polyacrylonitrile fiber formation. J. Appl. Polym. Sci. 2005, 96 (5), 1529-1533.

(19) Fink, H. P.; Weigel, P.; Purz, H. J.; Ganster, J. Structure formation of regenerated cellulose materials from NMMO-solutions. Prog. Polym. Sci. 2001, 26 (9), 1473-1524.

(20) Ago, M.; Endo, T.; Hirotsu, T. Crystalline transformation of native cellulose from cellulose I to cellulose II polymorph by a ballmilling method with a specific amount of water. Cellulose 2004, 11 (2), 163-167.

(21) Cheng, G.; Varanasi, P.; Arora, R.; Stavila, V.; Simmons, B. A.; Kent, M. S.; Singh, S. Impact of ionic liquid pretreatment conditions on cellulose crystalline structure using 1-ethyl-3-methylimidazolium acetate. J. Phys. Chem. B 2012, 116 (33), 10049-10054.

(22) Brown, R. M., Jr., Ed. Cellulose and other natural polymer systems: Biogenesis, structure, and degradation; Plenum Press: New York, U.S., 1983.

(23) O'Sullivan, A. Cellulose: the structure slowly unravels. Cellulose 1997, 4 (3), 173-207.

(24) Segal, L.; Creely, J. J.; Martin, A. E.; Conrad, C. M. An Empirical Method for Estimating the Degree of Crystallinity of Native Cellulose Using the X-Ray Diffractometer. Text. Res. J. 1959, 29 (10), 786-794.

(25) Park, S.; Baker, J.; Himmel, M.; Parilla, P.; Johnson, D. Cellulose crystallinity index: measurement techniques and their impact on interpreting cellulase performance. Biotechnol. Biofuels 2010, 3 (1), 10.

(26) Garvey, C. J.; Parker, I. H.; Simon, G. P. On the interpretation of X-ray diffraction powder patterns in terms of the nanostructure of cellulose I fibres. Macromol. Chem. Phys. 2005, 206 (15), 1568-1575.

(27) Cheng, G.; Varanasi, P.; Li, C.; Liu, H.; Melnichenko, Y. B.; Simmons, B. A.; Kent, M. S.; Singh, S. Transition of cellulose crystalline structure and surface morphology of biomass as a function of ionic liquid pretreatment and its relation to enzymatic hydrolysis. Biomacromolecules 2011, 12 (4), 933-941.

(28) Kim, J. T.; Netravali, A. N. Fabrication of advanced "green" composites using potassium hydroxide $(\mathrm{KOH})$ treated liquid crystalline (LC) cellulose fibers. J. Mater. Sci. 2013, 48 (11), 39503957.

(29) Das, M.; Chakraborty, D. Influence of alkali treatment on the fine structure and morphology of bamboo fibers. J. Appl. Polym. Sci. 2006, 102 (5), 5050-5056.

(30) Gedde, U. Polymer Physics; Springer: Netherlands, 1995.

(31) Hermans, P. H.; Hermans, P. Contribution to the physics of cellulose fibres: a study in sorption, density, refractive power and orientation; Elsevier: Amsterdam, Netherlands, 1946.

(32) Lafrance, C. P.; Pezolet, M.; Prud'homme, R. E. Study of the distribution of molecular orientation in highly oriented polyethylene by x-ray diffraction. Macromolecules 1991, 24 (17), 4948-4956.

(33) Mitchell, G. R.; Lovell, R. Application of cylindrical distributionfunctions to wide-angle X-ray-scattering from oriented polymers. Acta Crystallogr., Sect. A: Cryst. Phys., Diffr., Theor. Gen. Crystallogr. 1981, 37 (03), 189-196.

(34) Hermans, J. J.; Hermans, P. H.; Vermaas, D.; Weidinger, A. Quantitative evaluation of orientation in cellulose fibres from the X-ray fibre diagram. Recl. Trav. Chim. Pays-Bas 1946, 65 (6), 427-447.

(35) Northolt, M. G. Tensile deformation of poly (p-phenylene terephthalamide) fibers, an experimental and theoretical-analysis. Polymer 1980, 21 (10), 1199-1204.

(36) Zhang, H.; Wang, Z. G.; Zhang, Z. N.; Wu, J.; Zhang, J.; He, H. $\mathrm{S}$. Regenerated-cellulose/multiwalled-carbon-nanotube composite fibers with enhanced mechanical properties prepared with the ionic liquid 1-allyl-3-methylimidazolium chloride. Adv. Mater. 2007, 19 (5), 698-704.

(37) Michud, A.; Tanttu, M.; Asaadi, S.; Ma, Y.; Netti, E.; Kääriainen, P.; Persson, A.; Berntsson, A.; Hummel, M.; Sixta, H. Ioncell-F: ionic liquid-based cellulosic textile fibers as an alternative to viscose and Lyocell. Text. Res. J. 2016, 86, 543.

(38) Davies, R. J.; Montes-Moran, M. A.; Riekel, C.; Young, R. J. Single fibre deformation studies of poly(p-phenylene benzobisoxazole) fibres - Part II - Variation of crystal strain and crystallite orientation across the fibre. J. Mater. Sci. 2003, 38 (10), 2105-2115.

(39) Eichhorn, S. J.; Young, R. J.; Davies, R. J.; Riekel, C. Characterisation of the microstructure and deformation of high modulus cellulose fibres. Polymer 2003, 44 (19), 5901-5908.

(40) Kong, K.; Davies, R. J.; McDonald, M. A.; Young, R. J.; Wilding, M. A.; Ibbett, R. N.; Eichhorn, S. J. Influence of domain orientation on the mechanical properties of regenerated cellulose fibers. Biomacromolecules 2007, 8 (2), 624-630.

(41) Boerstoel, H. Self-organization phenomena in liquid crystal spinning. Sen'i Gakkaishi 2006, 62 (4), P93-P101.

(42) Lim, S. K.; Cho, K. M.; Tasaka, S.; Inagaki, N. Mesoporous carbon fibers prepared from regenerated rice straw fibers. Macromol. Mater. Eng. 2001, 286 (3), 187-190.

(43) Ashby, M. F. The CES EduPack database of natural and manmade materials; Cambridge University and Granta Design: Cambridge, UK, 2008; www.grantadesign.com/download/pdf/biomaterials.pdf. 
2016-07-27

High modulus regenerated cellulose

fibers spun from a low molecular weight microcrystalline cellulose solution

\section{Zhu, Chenchen}

American Chemical Society

Zhu C, Richardson RM, Potter KD, et al., (2016) High modulus regenerated cellulose fibers spun from a low molecular weight microcrystalline cellulose solution. ACS Sustainable

pÿChemistry and Engineering, Volume 4, Issue 9, 2016 pp. 45454553

http://dx.doi.org/10.1021/acssuschemeng.6b00555

Downloaded from Cranfield Library Services E-Repository 